\title{
Supremo Tribunal de la Signatura Apostólica. Sentencia*
}

Prot. N. 32372/01 C.A.

Dioecesis N.

Revocationis decreti poenalis ab Exc.mo Episcopo d. 6 Maii a. 1999 adversus Rev.dum X lati (Exc.mus Ordinarius Congregatio pro Clericis) in nomine Domini. Amen.

\section{SUPREMUM SIGNATURAE APOSTOLICAE TRIBUNAL}

Ioannis Pauli PP. II anno Summi Pontificatus XXVI, die 3 iulii 2004, videntibus Em.mis ac Rev.mis D.nis Antonio Maria Card. Rouco Varela, Eduardo Michaële Card. Egan, Petro Card. Erdö, Augustino Card. Cacciavillan, Ponente, Iuliano Card. Herranz, atque Exc.mis ac Rev.mis D.nis Augustino Vallini, Praefecto, Eduardo Davino, Henrico Mussinghoff, Francisco Coccopalmerio et Thoma Georgio Doran, intervenientibus Cl.mo C. Gullo tamquam Patrono Exc.mi Episcopi N., Cl.ma M. Wegan utpote Patrona Congregationis

* Traducción del Prof. Jordi Bosch Carrera.
Prot. N. 32372/01 C.A.

Diócesis N.

Sobre la revocación de un decreto penal dado por el Excmo. Obispo el día 6 de mayo de 1999 contra el Rvdo. X. de la diócesis N. (Excmo. Ordinario - Congregación para el Clero) en el nombre del Señor. Amén.

\section{EL SUPREMO TRIBUNAL DE LA SIGNATURA APOSTÓLICA}

El día 3 de julio de 2004, en el 26 año del Pontificado de Juan Pablo II, habiendo estudiado la cuestión los Emmos. y Rvdos. Sres. Cardenales Antonio Rouco Varela, Eduardo Michaele Egan, Peter Erdö, Agustín Cacciavillan como ponente, Julián Herranz y los Excmos. y Rvdos. Sres. Agustín Vallini, prefecto, Eduardo Davino, Enrique Mussinghoff, Francesco Coccopalmerio y Tomás Jorge Doran, y habiendo intervenido el abogado C. Gullo como patrono del Excmo. Obispo N., la abogada M. Wegan como patrona de la 
pro Clericis Rev.dique X et Rev.mo F. Daneels, o.praem., Promotore Iustitiae, in causa de qua supra, hanc tulit

\section{DEFINITIVAM SENTENTIAM}

\section{Facti species}

1. Rev.dus X a mense ianuario 1990 ad mensem novembrem 1995 parochus erat paroeciae $Z$ et dein parochus paroeciae $\mathrm{Y}$ in loco v.d. "W".

Suppressa paroecia $\mathrm{S}$ medio anno 1996, detectum est magnam pecuniae summam annis 1990-1996 in illis paroeciis acceptam et expensam competentem auctoritatem dioecesanam celatam fuisse atque de illius pecuniae usu saltem magna ex parte non haberi vel non amplius haberi ulla documenta vel adnotationes. Quam ob rem Exc.mus Episcopus N. illi presbytero die 20 februarii 1998 notificavit decisionem instituendi processum poenalem administrativum et die 30 decembris 1998 eum de accusationibus certiorem fecit.

Exhibita die 31 martii 1999 scripta defensione a Rev.do A pro Rev.do X, Exc.mus Episcopus die 6 maii eiusdem anni poenale tulit decretum (Summ., pp. 154-161), quo eum ob violationem can. 1377, aggravatam ob abusum officii (cfr. can. $1326 \S 1$, n. 2), privabat "of all rights, privileges and faculties granted by universal or particular law... Father $\mathrm{X}$ is, therefore, not authorized to exercise the ministry of the priesthood either publicly or privately in any manner whatsoever. In view of the amount of money which it has been demonstrated that Father $\mathrm{X}$ has alienated for his own purposes, he is also deprived of his right of remuneration, with due regard for the provision of $\mathrm{ca}-$ non $1350, \$ 1 ”$.
Congregación para el Clero y el Rvdo. X y el Rvdo. F. Daneels, o. praem., como Promotor de Justicia, en la causa, dio esta

\section{SENTENCIA DEFINITIVA}

\section{Hechos}

1. El Rvdo. X era párroco desde el mes de enero de 1990 hasta el mes de noviembre de 1995 en la parroquia $Z_{y}$ después párroco de la parroquia $Y$ en el lugar conocido como "W".

Una vez suprimida la parroquia $\mathrm{Z}$ a mediados de 1996, se descubrió que una gran suma de dinero, recibida y gastada durante los años 1990-1996 en aquellas parroquias, había sido ocultada a la autoridad competente diocesana; y que sobre el uso de ese dinero, al menos en gran parte, no había o más bien ya no hay justificante alguno. Por este motivo, el 20 de febrero de 1998, el Excmo. Obispo N. notificó a aquel presbítero la decisión de instituir un proceso penal administrativo y, el día 30 de diciembre de 1998, le comunicó las acusaciones.

Presentado el 31 de marzo de 1999 el escrito de defensa por parte del Rvdo. A en favor del Rvdo. X, el Excmo. Obispo, el día 6 de mayo del mismo año, emanó un decreto penal (Summ., pp. 154-161), por el que le privaba por violación del canon 1377 agravado por el abuso del oficio (cfr. can. $1326 \$ 1$, n. 2), de "todos los derechos, privilegios y facultades concedidas por el derecho universal y particular... El Padre X está, desde entonces, desautorizado para ejercer el ministerio sacerdotal tanto pública como privadamente de cualquier manera. En vista a la cantidad de dinero que, según ha sido demostrado, el Padre $\mathrm{X}$ ha gastado para sus propios intereses, él también ha sido privado de su derecho a remuneración, con respecto a la provisión del canon 1350, \$1”. 
Quae poena imponebatur ad tempus indefinitum, donec Rev.dus X recognosceret "his responsibility for all charges... and made full restitution...". Ad violationem vero can. 1373 quod attinet, una ex parte agnoscebatur hanc ob rem imponi non posse poenam, alia tamen ex parte sermo fiebat de circumstantia aggravanti et de necessitate pro remissione poenae etiam recognoscendi "charges arising from" can. 1373 .

2. Frustra petita revocatione praefati decreti (Summ., pp. 170-172), Patronus Rev.di X die 22 iunii 1999 recursum penes Congregationem pro Clericis instituit (Summ., pp. 184-190), quae die 13 martii 2001 (Summ., pp. 206-207) decisionem tulit in favorem Recurrentis, decernens ut ei restituerentur facultates ministeriales, ut revocarentur omnes aliae poenae ei impositae, utque ipse denuo ad ministerium pastorale in dioecesi exercendum admitteretur.

Exc.mus Episcopus tunc epistolis dierum 3 et 6 aprilis 2001 (Summ., pp. 208215) a Congregatione petiit ut decisionem suam revocare vel saltem emendare vellet, sed Congregatio die 26 aprilis 2001 hanc petitionem revocationis vel emendationis sui decreti reiecit, in epistola vero transmissionis indicans se removere quaestionem remunerationis Rev.do X compensandae (Summ., pp. 217-218), attentis litteris a Patrono Rev.di Recurrentis, die 10 aprilis 2001 ad Exc.mum Episcopum datis (Summ., p. 216).

3. Non acquievit Exc.mus Episcopus, qui die 27 aprilis 2001 ad H.S.T. provocavit (Summ., pp. 219-224; cfr. pp. 224225), censens recursum habere effectum suspensivum ad impugnatam Congregationis pro Clericis decisionem quod attinet (Summ., p. 227).
Esta pena se impuso por un tiempo indefinido, hasta que el Rvdo. X reconociera "su responsabilidad en todos estos cargos... y realizara la completa restitución...". En lo que se refiere a la violación del can. 1373 , por una parte, era sabido que no se podía imponer una pena por este motivo, por otra parte, se hacía mención de la circunstancia agravante y de la necesidad, para la remisión de la pena, del reconocimiento "de los cargos derivados del” can. 1373.

2. Suplicada en vano la revocación del mencionado decreto (Summ., pp. 170-172), el Patrono del Rvdo., el día 22 de junio de 1999 presentó un recurso ante la Congregación para el Clero (Summ., pp. 184-190), que el día 13 de marzo de 2001 (Summ., pp. 206-207) dio una decisión en favor del Recurrente, determinando que se le restituyeran las facultades ministeriales, se revocaran todas las penas que le habían sido impuestas y que fuera admitido finalmente al ejercicio del ministerio pastoral en la diócesis.

El Excmo. Obispo, mediante las cartas del 3 y 6 de abril de 2001 (Summ., pp. 208-215) pidió a la Congregación que revocara su decisión o, al menos, la enmendara. La Congregación, el día 26 de abril de 2001 rechazó esta petición de revocación o rectificación de su decreto, indicando, no obstante, en la carta de notificación del decreto, que se desmarcaba de la cuestión de la compensación económica al Rvdo. X (Summ., pp. 217-218), en atención a la carta del Patrono del Rvdo. Recurrente dada el 10 de abril de 2001 al Excmo. Obispo (Summ., p. 216).

3. El Excmo. Obispo no cejó en su empeño y el 27 de abril de 2001 acudió a este supremo Tribunal (Summ., pp. 219224; cfr. pp. 224-225), pensando que el recurso tenía efecto suspensivo con respecto a la decisión impugnada de la Congregación para el Clero (Summ., p. 227). 
Praemissa consueta discussione causae, recursus in Congressu diei 25 octobris 2002 ad disceptationem admissus est (Summ., p. 321) et dubium die 8 novembris 2002 hac sub formula concordatum (Summ., p. 322):

An constet de violatione legis in procedendo vel in decernendo relate ad decisionem Congregationis pro Clericis diei 13 Martii 2001, et, quatenus affirmative, num ab H.S.T. de damnis videndum sit.

Decreto diei 4 decembris 2002 Exc.mus H.S.T. Secretarius mandavit "Ill.mum D.num B 'Revisore-Contabile della Prefettura degli Affari Economici della Santa Sede' ut votum suum promere velit super quaestionibus oeconomicis de quibus in... causa" (Summ., p. 325). Quo voto die 15 septembris 2003 obtento (Summ., p. 326), Summarium causae confectum est.

Re denuo rite discussa inter Cl.mos Patronos Partium et Rev.mum Promotorem Iustitiae, Nobis dubio proposito respondendum est.

\section{In iure et in facto}

4. Impugnatum Congregationis pro Clericis decretum diei 13 martii 2001, ab eadem -expuncta vero quaestione de remuneratione Rev.di X compensanda- die 26 aprilis eiusdem anni confirmatum, in primis has memorat accusationes, de quibus in decreto poenali Exc.mi Episcopi N., scilicet: a) Rev.dum X sine requisita licentia bona ecclesiastica alienasse (can. 1377); b) ipsum inter fideles odium adversus Episcopum suscitasse (can. 1373) et c) ipsum alienasse "parish funds for his own purposes" seu reum esse s.d. "appropriationis indebitae" (Summ., p. 160, in fine).
Tenida la prevista discusión de la causa, el recurso fue admitido para su discusión en el Congreso del día 25 de octubre de 2002 (Summ., p. 321) y el dubio fue concordado el 8 de noviembre bajo esta fórmula (Summ., p. 322):

Si consta la violación de ley in procedendo o bien in decernendo en relación a la decisión de la Congregación para el Clero del 13 de Marzo de 2001, y, en caso afirmativo, si este Supremo Tribunal tiene que decidir sobre la reparación de daños causados.

Mediante decreto del 4 de diciembre de 2002, el Secretario de Este Supremo Tribunal ordenó "al Sr. B 'revisor-contable de la Prefectura de los Asuntos Económicos de la Santa Sede' que diera su parecer sobre las cuestiones económicas de esta causa" (Summ., p. 325). Obtenido su parecer el 15 de septiembre de 2003 (Summ., p. 326), fue elaborado el Sumario de la causa.

Discutida nuevamente la causa entre los Patronos de las Partes y el Rvdo. Promotor de Justicia, Nos corresponde responder al dubio propuesto.

\section{In iure e in facto}

4. El decreto impugnado del día 13 de marzo de 2001 de la Congregación para el Clero, confirmado por la misma -dejada a parte la cuestión de la compensación económica del Rvdo. X- el 26 de abril del mismo año, recoge en primer lugar estas acusaciones, que están en el decreto penal del Excmo. Obispo N., a saber: a) que el Rvdo. $\mathrm{X}$ sin la debida licencia enajenó bienes eclesiásticos (can. 1377); b) que él mismo suscitó odio entre los fieles contra el Obispo (can. 1373) y c) que él mismo vendió "fondos de la parroquia para sus propios intereses" o sea que fue reo de la así llamada "apropiación indebida" (Summ., p. 160, in fine). 
Ad secundam accusationem quod attinet, Dicasterium, cum ipse Exc.mus Episcopus in suo decreto poenali iam agnoverit non haberi ex can. 1373 sufficientes rationes ad poenam irrogandam, cum Exc.mo Episcopo consentit et proinde hac de re in suo decreto non amplius tractat.

Ad questionem de asserta "appropriatione indebita" quod attinet, Congregatio contendit quod: "after careful consideration... it cannot be concluded that the Rev. X had 'alienated parish funds for his own purposes', it could be concluded however that there was a certain amount of mismanagement and poor bookkeeping involved..., but criminal misappropiation of funds is not evident".

Ad violationem vero can. 1377 quod attinet, idem vero Dicasterium non dicit explicite eundem canonem applicari non posse in casu, quia requisita licentia, de qua in eodem canone, non requirebatur in assertis alienationibus a Rev.do X patratis. Rev.mus vero Promotor Iustitiae in voto suo diei 3 maii 2004 (pp. 6-9) abundanter exposuit delictum, de quo in can. 1377 , respicere facti speciem valde specificam et haudquaquam extendi posse ad alias violationes legis contra patrimonium personae iuridicae canonicae.

5. In iure, enim, res ita se habet:

- Praescriptum can. 1377 -scilicet: "Qui sine praescripta licentia bona ecclesiastica alienat, iusta poena puniatur"respicit cann. 1291 ss. et $638 \$ 3$, atqui in can. 1291 non agitur de qualibet alienatione, sed solummodo de illa quae spectat "bona, quae personae iuridicae publicae ex legitima assignatione patrimonium stabile constituunt et quorum valor summam iure definitam excedit" (cfr. J. T. Martín de Agar, Comentario exegético...,
En lo que se refiere a la segunda acusación, el Dicasterio -como el propio Excmo. Obispo, en su decreto penal, ya era consciente de no tener, según el can. 1373 , suficientes razones para irrogar una pena- tiene el mismo parecer que el Excmo. Obispo y por tanto sobre esta cuestión ya no trata más en su decreto.

En lo que se refiere a la cuestión de la afirmada "apropiación indebida", la Congregación afirma que: "después de un cuidadoso estudio... no se puede concluir que el Rvdo. X haya 'enajenado fondos de la parroquia para sus propios intereses', se podría concluir, sin embargo, que había una cierta proporción de mala gestión y mala contabilidad implicada en el tema..., pero no es evidente una malversación de fondos".

En lo que se refiere a la violación del can. 1377, el Dicasterio no dice explícitamente que el mismo canon no pueda ser aplicado en este caso, porque la debida licencia de la que habla el canon, no se requiere en las enajenaciones realizadas por el Rvdo. X. El Rvdo. Promotor de Justicia, en cambio, en su parecer del día 3 de mayo de 2004 (pp. 6-9) expone ampliamente que el delito del que se trata en el can. 1377 , contempla un hecho muy específico y no puede extenderse en modo alguno a otras violaciones de la ley contra el patrimonio de una persona jurídicocanónica.

5. Así pues, según el derecho, quedan así las cosas:

- La prescripción del can. 1377 -a saber: "Quien enajena bienes eclesiásticos sin la licencia prescrita, debe ser castigado con una pena justa"- mira a los cann. 1291 ss. y $638 \$ 3$, pero con todo el can. 1291 no trata de cualquier enajenación, sino solo de aquella que se refiere a "bienes que por asignación legítima constituyen el patrimonio estable de una persona jurídica pública y cuyo valor supera la cantidad establecida por el derecho" (cfr. 
ed. $1^{\mathrm{a}}$, IV/1, 524; K. LÜDICKE, MK, 1377/1, n. 2; Codice di Diritto Canonico commentato, Ancora, 2001, p. 1088).

- Disputatur inter auctores utrum, necne, can. 1377 sese quoque referat ad can. 1295, iuxta quem: "Requisita ad normam cann. 1291-1294, quibus etiam statuta personarum iuridicarum conformanda sunt, servari debent non solum in alienatione, sed etiam in quolibet negotio, quo condicio patrimonialis personae iuridicae peior fieri possit" (cfr. K. LÜDICKE, MK, 1377/1, n. 2; J. T. MARTÍN DE AGAR, Comentario exegético..., ed. 1 $1^{\mathrm{a}}, \mathrm{IV} / 1,524$, una ex parte, et V. DE PaOLIS - D. Cito, Le Sanzioni nella Chiesa, Urbaniana Univ. Press, p. 320, alia ex parte). Leges, enim quae poenam constituunt, "strictae subsunt interpretationi" (can. 18). Quidquid est, opinionum hac de re in doctrina diversitas permittit tenere quod in casu habetur dubium iuris, atqui "in dubio standum est pro reo".

- Doctrina, utcumque, interpretatur personae iuridicae condicionem patrimonialem, de qua in can. 1295, tamquam condicionem eiusdem patrimonii stabilis, quod quidem legitima assignatione determinatur. Quae assignatio "è un atto di straordinaria amministrazione... e va provata: la presunzione è che $\mathrm{i}$ beni non siano assegnati al patrimonio stabile. Alcuni beni, però, independentemente dalla legittima assegnazione, sono indisponibili..." (Codice di Diritto Canonico commentato, 2001, p. 1020: ad can. 1295). Et De Paolis (I beni temporali della Chiesa, Bologna 1995, p. 193) animadvertit quod: "Il denaro per sé non appartiene al patrimonio stabile, a meno che non sia vincolato stabilmente come parte del patrimonio stabile".

- Violatio can. 1377 proinde haberi nequit, nisi agatur de alienatione vel, si et quatenus, de negotiis assimilatis, quae pa-
J. T. Martín de Agar, Comentario exegético..., ed. $1^{\text {a }}$, IV/1, 524; K. LÜDICKE, MK, 1377/1, n. 2; Codice di Diritto Canonico commentato, Ancora, 2001, p. 1088).

- Los autores discuten si el c. 1377 se refiere también al can. 1295, según el cual: "los requisitos establecidos en los cann. 1291-1294, a los que también se han de acomodar los estatutos de las personas jurídicas, deben observarse no solo en una enajenación, sino también en cualquier operación de la que pueda resultar perjudicada la situación patrimonial de la persona jurídica" (cfr. K. LÜDICKE, MK, 1377/1, n. 2; J. T. MARTÍN DE AgAR, Comentario exegético..., ed. $1^{\text {a }}$, IV/1, 524, por una parte, y V. DE PAOLIS - D. Cito, Le Sanzioni nella Chiesa, Urbaniana Univ. Press, p. 320, por otra). Las leyes que establecen una pena, "se deben interpretar estrictamente" (can. 18). Sea lo que fuere, la diversidad de opiniones sobre esta cuestión permite sostener que en este caso nos encontramos ante un dubium iuris, así que "en la duda hay que estar a favor del acusado".

- La doctrina, en todo caso, entiende la condición patrimonial de la persona jurídica, de la que habla el can. 1295, como la condición del mismo patrimonio estable. Esta asignación "es un acto de administración extraordinaria... $\mathrm{y}$ ha de ser probada: la presunción es que los bienes no estén asignados al patrimonio estable. Algunos bienes, en cambio, independientemente de la legítima asignación, son indisponibles" (Codice di Diritto Canonico commentato, 2001, p. 1020: ad can. 1295). Et De Paolis (I beni temporali della Chiesa, Bologna 1995, p. 193) señala que: "el dinero de por sí, pertenece al patrimonio estable, a no ser que esté vinculado establemente como parte del patrimonio estable".

- La violación del can. 1377, por tanto, no puede darse, sino se trata de una enajenación o bien, sino se trata de opera- 
trimonium stabile respiciunt et praeterea per se simul summam minimam a Conferentia Episcoporum statutam superant (cfr. can. 1292).

6. Item appropriatae et cogentes dicendae sunt Rev.mi Promotoris Iustitiae animadversiones in facto (cfr. votum eius, pp. 10-12):

- Exc.mus Episcopus contra obiectiones Rev.di A censuit cann. 1377 et 1295 esse ad rem in casu (Summ., p. 156) et hac in opinione, epistola die 15 novembris 1999 ad Congregationem data, institit (Summ., p. 194).

- Idem autem Exc.mus Praesul in suo decreto poenali nonnullas graves irregularitates a Rev.do X patratas adduxit, sed non satis indicavit quibus concretis Rev.di $\mathrm{X}$ illegitimis negotiis, quo modo et qua mensura condicio patrimonialis illarum paroeciarum inde reapse peior fieri potuisset.

- Ipse, praesertim, non attulit ullam alienationem a Rev.do X factam, ad quam specifica licentia iuxta cann. 1291 ss. requisita erat, sed ex asserta violatione can. 1295 dubium gressum fecit ad assertam violationem can. 1377, dum irregularitates adductae respiciunt pecuniam paroeciarum praesentem, quae nullo modo illarum paroeciarum patrimonio stabili assignata ex actis probatur; tota summa denique, de qua in casu, infra summam mansit minimam a Conferentia Episcoporum Statuum Foederatorum Americae Septentrionalis iuxta can. 1292 definitam (500.000 lib. SFAS).

7. Impugnatum Congregationis pro Clericis decretum, etsi non indicat modo explicito rationem motivam ob quam Rev.di X condemnationem poenalem ex can. 1377 sustineri nequit, eam nihilominus sustineri non posse censet, uti ipsa de facto sustineri nequit. Aliis verbis: impugnatum decretum in facti specie $(\$ \$ 2$ et 4$)$ ciones asimiladas que afectan al patrimonio estable y además de por si superan todas ellas la suma mínima establecida por la conferencia Episcopal (cfr. can. 1292).

6. Asimismo hay que decir que las animadversiones in facto del Rev. Promotor de Justicia son apropiadas y concluyentes (cfr. su dictamen, pp. 10-12):

- El Obispo contra las objeciones del Rev. A pensó que los cann. 1377 y 1295 eran adecuados al caso (Summ., p. 156) y con esta opinión, insistió en la carta enviada a la Congregación el 15 de noviembre de 1999 (Summ., p. 194).

- El mismo Prelado en su decreto penal adujo algunas graves irregularidades cometidas por el Rev. X, pero no indicó suficientemente mediante qué ilegítimas operaciones del Rev. X, de qué manera y en qué medida la condición patrimonial de aquellas parroquias pudiera haber ido a peor.

- Él, sobre todo, no aportó ninguna enajenación hecha por el Rev. X, para la cual fuera requerida una específica licencia según los cann. 1291 y ss., sino que por la reivindicada violación del can. 1295 se abrió paso a la duda hacia la violación del can. 1377, mientras que las irregularidades aducidas se refieren a la cantidad de dinero presente de las parroquias, que de ninguna manera está probado, por los hechos que constan en actas, que esté asignado al patrimonio estable de aquellas parroquias; la cual, en este caso, permanece por debajo de la cantidad mínima definida por la Conferencia episcopal de los Estados Unidos según el can. 1292 (500.000 lib. SFAS).

7. El decreto impugnado de la Congregación para el Clero, aunque no indica de manera explícita el motivo por el cual no se le puede mantener la condena penal al Rvdo. X según el can. 1377, sostiene que esta no puede ser mantenida en modo alguno, como de hecho no pudo ser mantenida. Con otras palabras: el decreto 
mentionem facit de accusatione violationis can. 1377 necnon de relativa poena ab Exc.mo Episcopo ob violatum can. 1377 imposita, sed dein concludit decernendo ut Exc.mus Episcopus omnes tollat poenas Rev.do X impositas, id quod significat Congregationem pro Clericis imputabilitatem eiusdem presbyteri pro delicto, de quo in can. 1377 , haudquaquam retinere.

Iam satis de condemnatione poenali ab Exc.mo Episcopo adversus Rev.dum X ex can. 1377 lata, quae in Congregationis pro Clericis decreto legitime sine valido fundamento habita est.

8. Legitur in fine decreti poenalis Exc.mi N. Episcopi diei 6 Maii 1999 (Summ., p. 161): "The penalties specified in this Decree are imposed for an indefinite period of time and will remain effective and in force until such time as Father $\mathrm{X}$ has [a] formally acknowledged his responsibility for all charges arising both from canons 1377 and 1373 and [b] made full restitution to the Diocese $N$. for all funds misappropriated from $\mathrm{Z}$ and $\mathrm{Y}$ parishes".

Ad rem impugnatum Congregationis decretum diei 13 Martii 2001 in parte motiva animadvertit agi de violatione can. $1728 \$ 2$ : "whereas penalties were imposed upon the Rev. X contingent upon his admission to the charges which were made against him in contravention of canon $1728 \$ 2 ”$.

Exc.mus vero Episcopus in epistola die 6 aprilis 2001 ad Congregationem data (Summ., pp. 197-198; cfr. p. 220) replicavit se non requisisse a Rev.do $\mathrm{X}$ delicti confessionem ad poenam irrogandam, sed tantum ad poenam remittendam (S. 197-198; 220).

Attamen, etiam hoc in casu, perpenso quod agitur de remissione poenae expiatoriae, requisita confessio congruere non videtur cum spiritu can. $1728 \$ 2$, iuxta impugnado, en sus facti species (\$\$2 y 4) menciona la acusación de violación del can. 1377 así como la correspondiente pena impuesta por el Obispo por la violación de ese canon, pero finalmente in decernendo concluye que el Excmo. Obispo debe retirar todas las penas impuestas al Rvdo. X, lo que significa que la Congregación para el Clero no sostiene la imputabilidad del mencionado presbítero en cuanto al delito del can. 1377.

Con todo esto queda claro el tema de la condena penal impuesta al Rvdo. X por el Obispo en virtud del can. 1377 que el decreto de la Congregación para el Clero tiene como carente de válido fundamento.

8. Al final del decreto penal del Excmo. Obispo N. del día 6 de mayo de 1999 (Summ., p. 161) se lee: "Las sanciones especificadas en este decreto se imponen por un periodo indefinido de tiempo y seguirán vigentes y en vigor hasta el momento en que el padre $\mathrm{X}$ [a] haya reconocido formalmente su responsabilidad por todos los cargos que se derivan de los cánones 1377 y 1373 y [b] haya hecho restitución total a la diócesis $\mathrm{N}$. de todos los fondos apropiados de las parroquias $\mathrm{Z}$ e $\mathrm{Y}$ ".

Con relación a este asunto, el decreto impugnado de la Congregación del día 13 de marzo de 2001, en la parte motiva, advierte que se trata de una violación del can. $1728 \$ 2$ : "las sanciones que se impusieron al Rev. X están supeditadas a su admisión de los cargos que se hicieron contra él por violación del canon $1728 \$ 2$ ".

El Excmo. Obispo en la carta del 6 de abril de 2001 enviada a la Congregación (Summ., pp. 197-198; cfr. p. 220) respondió que él no pidió del Rvdo. X la confesión del delito para irrogar la pena, sino solo para su remisión (S. 197-198; 220).

Sin embargo, también en este caso, teniendo en cuenta que se trata de la remisión de una pena expiatoria, la requerida confesión no parece congruente con el espíritu 
quem accusatus "ad confitendum delictum non tenetur".

9. Quibus dictis, iam patet non constare de violatione legis sive in procedendo sive in decernendo ad decisionem a Congregatione pro Clericis die 13 martii 2001 latam quod attinet. Cadit itaque altera pars dubii propositi, scilicet: "et quatenus affirmative, num ab H.S.T. de damnis videndum sit".

Si Exc.mus vero N. Episcopus instet pro restitutione, ipse rem Tribunali Metropolitano... (cfr. can. $1419 \$ 2$ ) deferat, nisi quaestio per arbitratum ad normam cann. 1713-1716 solvi possit.

10. Em.mi et Exc.mi H.S.T. Patres tamen animadvertunt Rev.do X difficulter denuo curam paroeciae committi posse (cfr. cann. 1740-1741) atque, si et quatenus, perpendendum esse num adversus eum, si recidivus fuerit, servato can. 1362, ex can. 1389 procedi possit.

\section{Conclusio}

11. Omnibus sive in iure sive in facto aeque rimatis, Collegium Em.morum atque Exc.morum Patrum pro Tribunali sedens ac solum Deum prae oculis habens, dubio proposito respondendum censuit uti respondet:

Negative, seu non constare de violatione legis in procedendo vel in decernendo relate ad decisionem Congregationis pro Clericis diei 13 Martii 2001;

Ad alterum: non proponi.

Et ad mentem.

Mens autem haec est: Episcopus uti potest, si et quatenus, iure suo, vi cann. 1740; 1741; 1389 C. I. C. del can. 1728 \$2, según el cual el acusado "no está obligado a confesar el delito".

9. Con estas consideraciones, resulta patente que no consta la violación de la ley, ya sea in procedendo ya sea in decernendo en lo que se refiere a la decisión tomada por la Congregación para el Clero del día 13 de marzo de 2001. Queda pendiente la segunda parte del dubio propuesto, a saber: "y en la medida en que sea afirmativo, si debe ser examinado por este Tribunal la cuestión de los daños".

Si el Excmo. Obispo N. pide la restitución, él mismo lleve la cuestión al Tribunal Metropolitano... (cfr. can. 1419 \$ 2), a no ser que la cuestión pueda ser resuelta mediante arbitraje según los cann. 1713-1716.

10. Los Emmos. y Excmos. Padres de este Tribunal advierten, sin embargo, que difícilmente puede confiársele al Rvdo. X la atención de la parroquia de nuevo (cfr. cann. 1740-1741) y, si el caso lo requiere, habría que valorar si, en caso de que fuera reincidente, pudiera procederse por el can. 1389, quedando a salvo el can. 1362.

\section{Conclusión}

11. Examinadas todas las cosas tanto in iure como in facto, el Colegio de los Emmos. y Excmos. Padres en la sesión de este Tribunal y con la mirada puesta solo en Dios, decidió responder al dubio propuesto como responde:

Negativamente, es decir, no consta violación de la ley in procedendo o in decernendo en relación con la decisión de la Congregación para el Clero del día 13 de marzo de 2001;

$\mathrm{Al}$ segundo dubio: no sea propuesto

Según la mente.

La mente es esta: El Obispo puede usar, en la medida que corresponda de su derecho en virtud de los cann. 1740; 1741; 1389 C. I. C. 
Summa ab Exc.mo Recurrente apud arcam Signaturae Apostolicae deposita retineatur pro expensis huius Supremi Tribunalis. Partes vero suo quaeque Cl.mo Patrono congruum solvant honorarium.

Ita pronuntiamus ac statuimus, mandantes iis quorum interest, ut hanc Nostram definitivam sententiam exsecutioni tradant, ad omnes iuris effectus.

Datum Romae, e sede Supremi Signaturae Apostolicae Tribunalis, die 3 iulii 2004.

+ Augustinus Vallini, Praefectus

Antonius Maria Card. Rouco Varela

Eduardus Michaël Card. Egan

Petrus Card. Erdö

Augustinus Card. Cacciavillan, Ponens

Iulianus Card. Herranz

+ Eduardus Davino

+ Henricus Mussinghoff

+ Franciscus Coccopalmerio

+ Thomas Georgius Doran

Die 21 octobris 2004

Notificetur.

+ Velasius De Paolis, Secretarius

Vicentius Cárcel Ortí, Moderator Cancellariae
Reténgase la cantidad depositada en las arcas de la Signatura Apostólica por el Excmo. Recurrente, para el pago de los gastos de este Supremo Tribunal. Que las partes paguen a sus patronos los correspondientes honorarios.

Así lo pronunciamos y lo establecemos, ordenando a los interesados que entreguen esta Nuestra sentencia definitiva para su ejecución a todos los efectos del derecho.

Dado en Roma, desde la sede del Supremo Tribunal de la Signatura Apostólica el 3 de julio de 2004.

+ Agustín Vallini, Prefecto

Antonio Maria Card. Rouco Varela

Eduardo Miguel Card. Egan

Pedro Card. Erdö

Augustín Card. Cacciavillan, Ponens

Julián Card. Herranz

+ Eduardo Davino

+ Enrique Mussinghoff

+ Francisco Coccopalmerio

+ Tomás Jorge Doran

En el 21 de octubre de 2004

Notifíquese

+ Velasio De Paolis, Secretario

Vicente Cárcel Ortí, Moderador de la Cancillería 